\title{
Segmentation of Highly Lignified Zones in Wood Fiber Cross-Sections
}

\author{
Bettina Selig ${ }^{1}$, Cris L. Luengo Hendriks ${ }^{1}$, Stig Bardage ${ }^{2}$, \\ and Gunilla Borgefors ${ }^{1}$ \\ 1 Centre for Image Analysis, \\ Swedish University of Agricultural Sciences, Box 337, \\ SE-751 05 Uppsala, Sweden \\ \{bettina, cris, gunilla\}@cb.uu.se \\ 2 Department of Forest Products, \\ Swedish University of Agricultural Sciences, Vallvägen 9 A-D, \\ SE-750 07 Uppsala, Sweden \\ Stig.Bardage@sprod.slu.se
}

\begin{abstract}
Lignification of wood fibers has important consequences to the paper production, but its exact effects are not well understood. To correlate exact levels of lignin in wood fibers to their mechanical properties, lignin autofluorescence is imaged in wood fiber cross-sections. Highly lignified areas can be detected and related to the area of the whole cell wall. Presently these measurements are performed manually, which is tedious and expensive. In this paper a method is proposed to estimate the degree of lignification automatically. A multi-stage snake-based segmentation is applied on each cell separately. To make a preliminary evaluation we used an image which contained 17 complete cell cross-sections. This image was segmented both automatically and manually by an expert. There was a highly significant correlation between the two methods, although a systematic difference indicates a disagreement in the definition of the edges between the expert and the algorithm.
\end{abstract}

\section{Introduction}

\section{$1.1 \quad$ Background}

Wood is composed of cells that are not visible to the naked eye. The majority of wood cells are hollow fibers. They are up to $2 \mathrm{~mm}$ long and $30 \mu \mathrm{m}$ in diameter and mainly consist of cellulose, hemicellulose and lignin [1]. Wood fibers are composed of a cell wall and a empty space in the center which is called lumen (see Fig. 1). The middle lamellae occupies the space between the fibers and contains lignin, which binds the cells together. Lignin also occurs within the cell walls and gives them rigidity 12 .

The process of lignin diffusion into the cell is called lignification: Lignin precursors diffuse from the lumen to the cell wall and middle lamellae. They condensate (lignificate) starting at the middle lamellae into the cell wall. A so-called condensation front arises (see Fig. 2) that separates the highly lignified zone from the normally lignified zone [2]. 


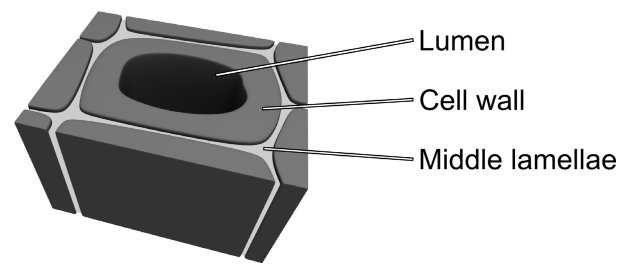

Fig. 1. A wood cell consists of two main structures: lumen and cell wall. The middle lamellae fills the space between the fibres.
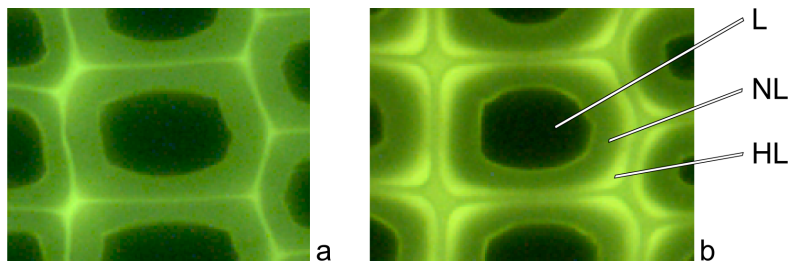

Fig. 2. Cross-section of a normal lignified wood cell (a) and a wood cell with highly lignified zone (b). The area of the lumen (L), the normally lignified zone (NL) and the highly lignified zone (HL) are well-defined in the autofluorescence microscope images. The boundary between NL and HL is called condensation front.

The effects of high lignification on the mechanical properties of wood fibers are especially important in paper production, but are not well understood. A high amount of lignin in the fibers causes bad paper quality. To study these effects it is necessary to measure the distribution of lignin throughout the fiber.

Because lignin is autofluorescent [3], it is possible to image a wood section in a fluorescence microscope with little preparation. The areas of lumen (L), normal lignified cell wall (NL) and highly lignified cell wall (HL) have to be identified so that they can be measured individually. The aim is to relate HL to the area of the whole cell wall. Presently this is done manually, but manual measurements are tedious, expensive and non-reproducible. To our knowledge there exists no automatic method to determine the size of HL in the cell wall. Therefore we are developing a proceeding to analyze large amounts of wood fiber cross-sections automatically. The resulting program will be used by wood scientists.

In fluorescence images edges are in general not sharp. This complicates the boundary detection seriously. Additionally, the condensation front is fuzzy nature and the boundaries around the cell walls have very low contrast at some points, which makes the detection by thresholding impossible.

\subsection{Active Contour Models}

Active contour models 4,5], known as snakes, are often used to detect the boundary of an object in an image especially when the boundary is partly missing or partly difficult to detect. After an initial guess, the snake $v(s)$ is deformed 
according to an energy function and converges to local minima which correspond mainly to edges in the image. This energy function is calculated from so called internal and external forces.

$$
E_{\text {snake }}=E_{\text {int }}+E_{\text {ext }}
$$

The internal force defines the most likely shape of the contour to be found. Its parameters, elasticity $\alpha$ and rigidity $\beta$, have to be well chosen to achieve a good result.

$$
E_{i n t}=\alpha\left|\frac{d v}{d s}\right|^{2}+\beta\left|\frac{d^{2} v}{d s^{2}}\right|^{2}
$$

The external force moves the snake towards the most probable position in the image. There exist many ways to calculate the external force. In this paper we use traditional snakes, in which the external force is based on the gradient magnitude of the image $I$. Therefore, regions with a large gradient attract the snakes.

$$
E_{\text {ext }}=-|\nabla I(x, y)|^{2}
$$

A balloon force is added that forces the active contour to grow outwards (towards the normal direction $\vec{n}(s)$ ) like a balloon [6]. This enables the snake to overcome regions where the gradient magnitude is too small to move it.

$$
F_{\text {ext }}=-\kappa \frac{\nabla E_{\text {ext }}}{\left\|\nabla E_{\text {ext }}\right\|}+\kappa_{p} \vec{n}(s)
$$

The difficulty with using active contour models lies in finding suitable weights for the different forces. The snake can get stuck in an area with a low gradient if the balloon force is too weak, or the active contour will overshoot the desired boundary if the balloon force is too strong compared to the traditional external force.

In section 2.2 a method is proposed, that considers the mentioned difficulties and expands the snake-based detection in order to find and segment the different regions of highly lignified wood cells in fluorescence light microscopy images.

\section{Materials and Methods}

\subsection{Material}

A sample of approximately $2 \times 1 \times 1 \mathrm{~cm}^{3}$ was cut from a wood disk of Scots pine, Pinus sylvestris L., and sectioned using a sledge microtome. Afterwards, transverse sections $20 \mu \mathrm{m}$ thick were cut and transferred onto an objective glass, together with some drops of distilled water, and covered with a cover glass. The images were acquired using a Leica DFC490 CCD camera attached to an epifluorescence microscope.

The acquired images have $1300 \times 1030$ pixels and a pixel size of $0.3 \mu \mathrm{m}$. Only the green channel was used for further processing, as the red and blue channels contain no additional information. 
In this paper we illustrate the proposed algorithm using an image section of $340 \times 500$ pixels shown in Fig. 3. This section contains representative cells with high lignification. An expert on wood fiber properties segmented manually 17 cells from this image for comparison.

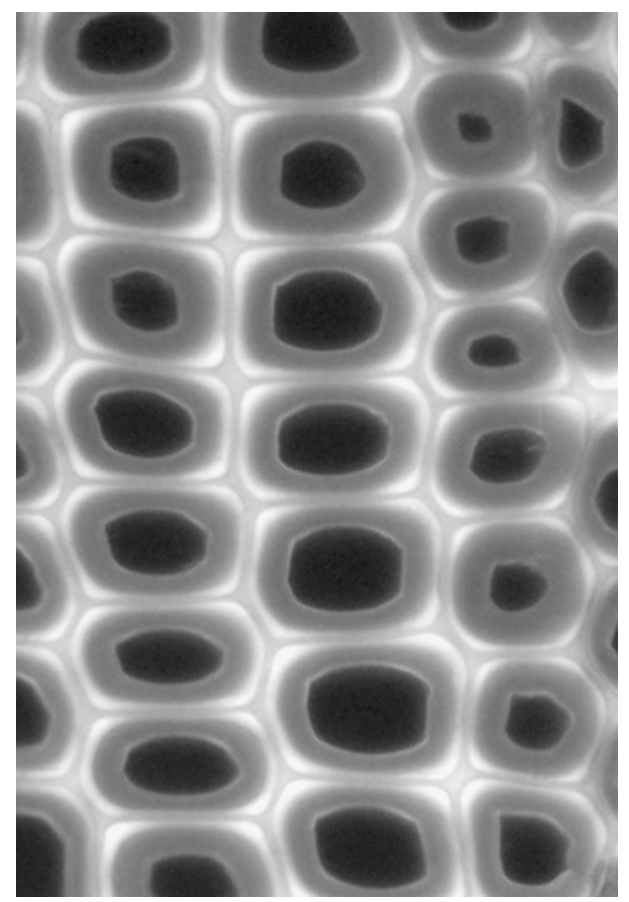

Fig. 3. Sample image with 17 representative cells used to illustrate the proposed algorithm

\subsection{Method}

The segmentation of the different regions is performed individually for each cell. The lumen is used as seed area for the snake-based segmentation. By expanding the active contour the relevant regions can be found and measured.

Finding Lumen. The lumen of a cell is significantly darker than the cell wall and the middle lamellae. This makes it possible to detect the lumens using a suitable global threshold. However, the histogram gives little help in determining an appropriate threshold level. Therefore we used a more complex automatic method based on a rough segmentation of the image by edges, yielding a few sample lumens and cell walls. These sample regions were then used to determine the correct global threshold level. The rough segmentation was accomplished as follows. 


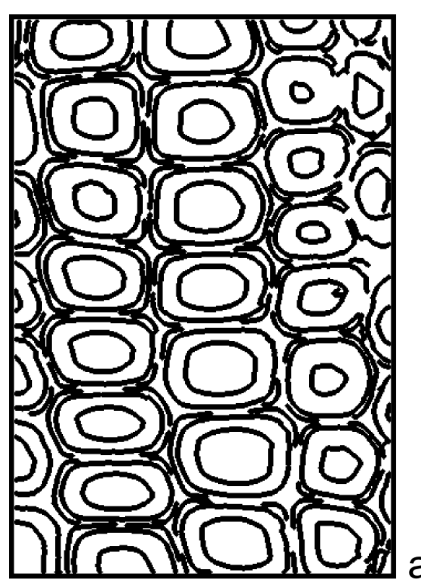

(a) Edge map

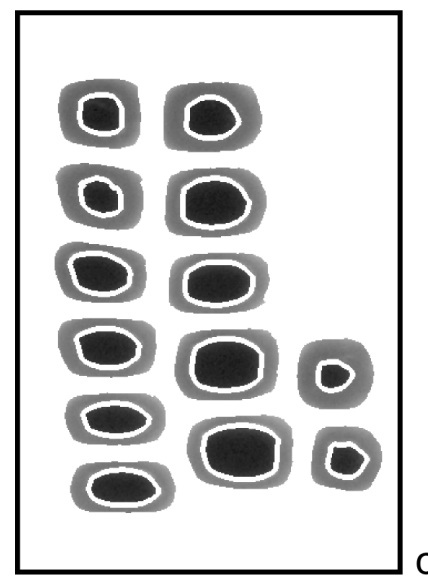

(c) Sample set of lumens and (d) All lumens after windowing cell walls

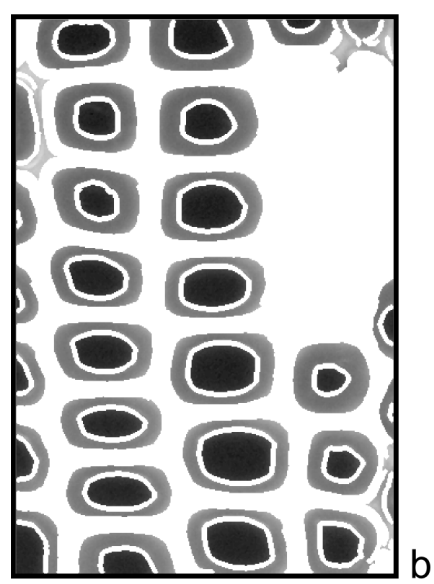

(b) Set of regions surrounded by another region

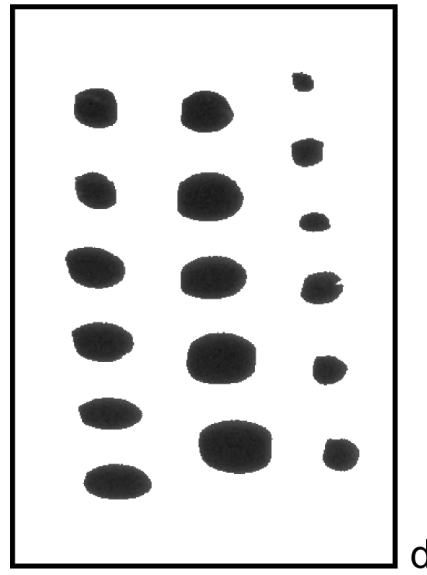

d

Fig. 4. Steps followed to find the fiber lumens in the image of Fig. 3

The Canny edge detector 7 followed by a small dilation yields a continuous boundary for most lumens and many of the cell walls (Fig. 4(a) . Each of these closed regions is individually labeled. Because a lumen is always surrounded by a cell wall, we now look for regions that are completely surrounded by another region (Fig. 4(b) . To avoid misclassification, we further constrain this selection to outer regions that are convex (the cross-section of a wood fiber is expected to be convex).

We now have a set of sample lumens and corresponding cell wall regions (Fig. 4(c)). The gray values from only these regions are compiled into a histogram, which typically is nicely bimodal with a strong minimum between the 
two peaks. This local minimum gives us a threshold value that we apply to the whole image, yielding all the lumens.

Only cells which are completely inside the image are useful for measurement purposes. To discard partial cells we define a square window surrounding the sample cell walls found earlier. The lumens that are not completely inside this window are discarded. The remaining lumen contours are refined using a snake with the traditional external force (Fig. 4(d).

The idea is to grow the snakes outwards to find the different regions of the cells successively. The segmentation is divided into three steps: Adapting a reasonable shape for the lumen boundary, locating the condensation front, and detecting the boundary between cell wall and middle lamellae.

We used the in [5] provided implementation of snakes with the parameters shown in Table 1 .

Table 1. Parameters used for the implementation of the algorithm, where $\alpha$ is elasticity and $\beta$ rigidity for the internal force, $\gamma$ viscosity (weighting of the original position), $\kappa$ the weighting for the external force and $\kappa_{p}$ the weighting for the balloon force. The parameters were chosen to work well on the test image, but the exact choices are not so important because a range of values produce nearly identical results.

\begin{tabular}{|l|c|c|c|c|c|}
\cline { 2 - 6 } \multicolumn{1}{c|}{} & $\alpha$ & $\beta$ & $\mathrm{V}$ & $\mathrm{K}$ & $\mathrm{K}_{\mathrm{p}}$ \\
\hline Boundary of L & 0.05 & 3 & 1 & 3 & 0.1 \\
\hline Intermediate step 1 & 0.05 & 3 & 1 & 3 & 0.1 \\
\hline Boundary of NL & 0.05 & 3 & 1 & 3 & 0.1 \\
\hline Intermediate step 2 & 0.05 & 3 & 1 & 3 & 0 \\
\hline Boundary of $\mathrm{HL}$ & 0.05 & 3 & 20 & 3 & 0.1 \\
\hline
\end{tabular}

After initializing the snake with the contour of the lumen found through thresholding, we apply a traditional external force (combined with a small balloon force). While pushing the snake towards the highest gradient, we refine the position of the lumen boundary.

Finding condensation front. The result from the first step is used as a starting point for the second step. Since the lumen boundary and the condensation front are very similar (both edges have the same polarity) it is impossible to push the snake away from the first edge and at the same time make sure it settles at the second edge. To solve this problem we use an intermediate step with a new external energy, which has its minima in regions with a small gradient magnitude.

$$
E_{1}=+|\nabla I(x, y)|^{2}
$$

Combined with a small balloon force, the snake converges to the region with the lowest gradient between the two edges. From this point, the condensation front can be found with a snake using a small balloon force and the traditional external force. 
Finding cell wall boundary. To locate the boundary between the cell wall and the middle lamellae a similar two-stage snake is applied. This time an external energy is used which has its minima in the areas with high gray values.

$$
E_{2}=-I(x, y)
$$

Since the highly lignified zones are very bright, the snake will converge in the middle of these regions. Afterwards, a traditional external force is used to push the snake outwards to detect the boundary between cell wall and middle lamellae.

Typically traditional snakes do not terminate. However, due to the combination of the chosen forces all snakes described in this paper converge to their final position after 10-20 steps. Afterwards only little changes occur and the algorithm is stopped after 30 steps.

\section{$3 \quad$ Results}

To make a preliminary evaluation of the proposed method we used an image which contained 17 detectable wood cells. This image was segmented independently by the proposed algorithm and manually by an expert. This delineation was performed after the algorithm was finished, and it was not used to define the algorithm. The regions L, NL and HL were measured and compared.

The results from the two analyses and the area HL related to the whole cell wall are compared in Fig. 6. Here, the horizontal axis represent the results from the automatic method and the vertical axis from manual measurements. The solid line in the figure is the identity. Measurements on this line had the same result in the manual and automatic method. Values left of this line were underestimated by the proposed algorithm and values right of this line were overestimated.

The area of the lumen was measured well, whereas NL was a bit overestimated and HL generally underestimated.

The relative area of the highly lignified zone was computed as $p=\frac{H L}{N L+H L}$. These results reflect the overestimated measurements of HL.
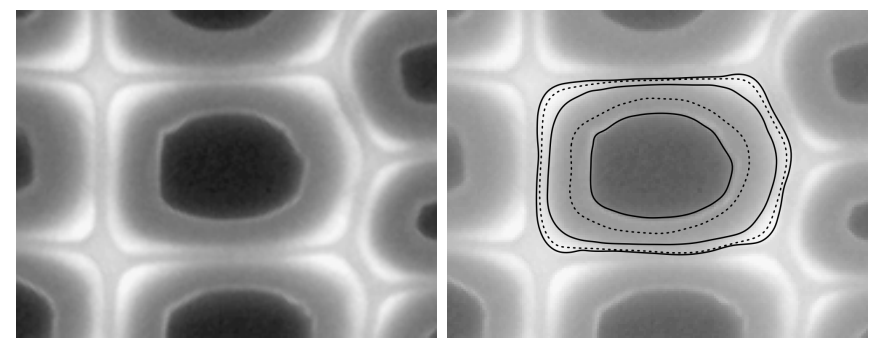

Fig. 5. Final result for one wood cell (solid lines) with intermediate steps (dotted lines) 


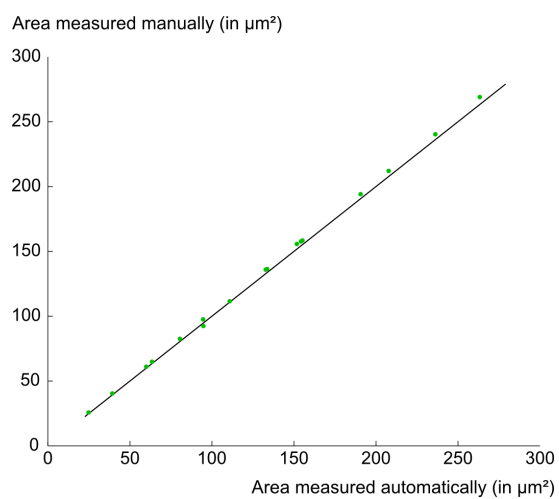

(a) Size of area L

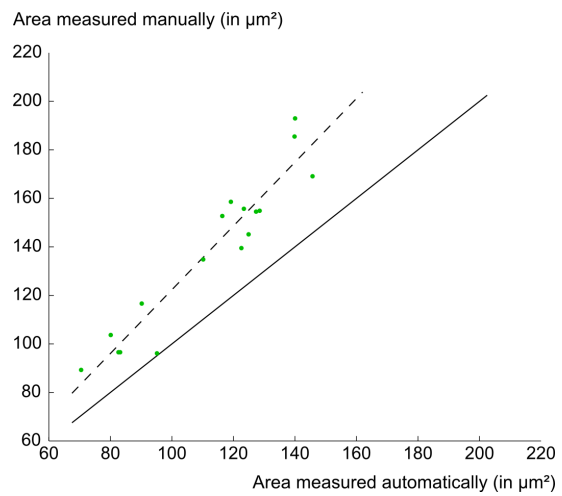

(c) Size of area HL

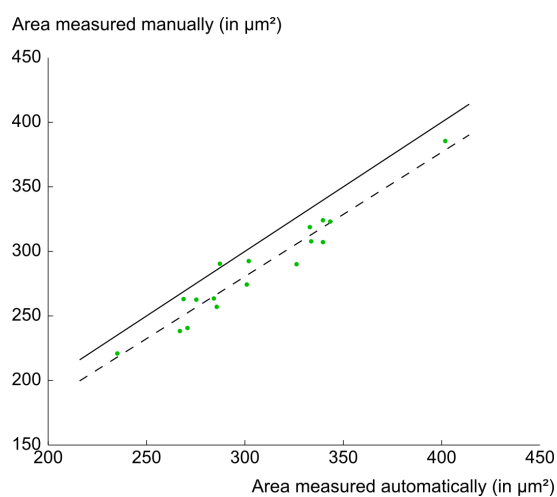

(b) Size of area NL

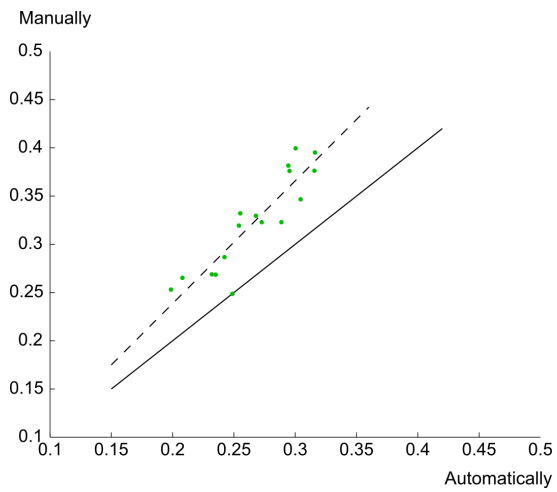

(d) Size of HL in relation to size of the whole cell wall.

Fig. 6. Comparison between manual and automatic method

\section{Discussion and Conclusions}

The automatic labeling and the expert agreed to a different degree for each of the boundaries. These disparities have various reasons.

First of all, manual measuring is always subjective and not deterministic. The criteria used can differ from expert to expert, as well as within a series of measurements performed by a single expert. The boundaries can be drawn inside, outside or directly on the edge. The proposed algorithm sets the boundaries on the edges, whereas our expert places them depending on the type of boundary. For example, the lumen boundary was consistently drawn inside the lumen, and the outer cell boundary outside the cell. In short, the expert delineated the cell wall rather than marking its boundary. It can be argued that for further automated quantification of lignin it is more valuable to have identified the boundaries between the regions. In Figure 7 you can see an example of the boundary of HL done both automatically and manually. Here it is apparent 


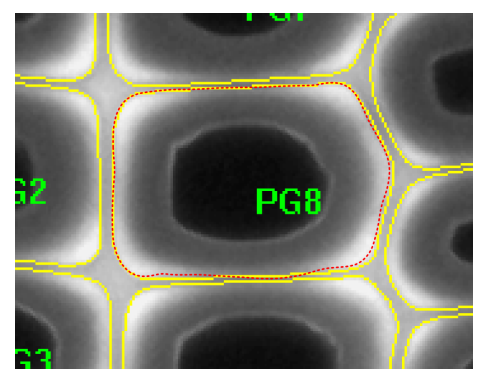

Fig. 7. Manual (solid line) and automatic (dotted line) segmentation of the outer boundary of a cell

that the manually placed boundary lies outside the one created by the proposed algorithm.

Although the results of HL do not follow the identity line, they are scattered around a (virtual) second line which is slightly tilted and shifted relative to the identity. This systematic error shows that even though the measurements followed slightly different criteria a close relation exists.

Another characteristic of the edges can be detected in the result graphs. The region NL has blurry and fuzzy boundaries and the edges around HL have very low contrast at some points. Both are difficult to detect either manually or automatically. Therefore, the plots for these boundaries show a larger degree of scatter then the highly correlated plot of L. The lumen has a sharp and well defined boundary that allows for a more precise measurement. But in spite of everything, the calculated correlation is high for all the regions (see Table 2).

Table 2. Correlation between manual and automatic measurements of the areas L, NL and HL. (All the p-values are less than $10^{-8}$ ).

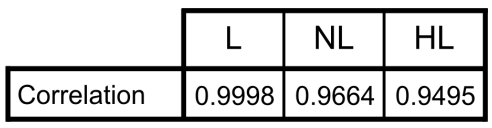

We tested the algorithm on other images and obtained similar results. In this paper we show the algorithm applied to this one particular image because that is the one we have a manual segmentation for and therefore it's the only data we have we can do comparisons on.

Currently the algorithm is applied on each cell separately. An improvement will be to grow regions simultaneously, allowing them to compete for space (e.g. 8] ). This would be particularly useful when segmenting not highly lignified cells, because for these cells the current algorithm is not able to distinguish the edges, producing overlapping regions. 


\section{References}

1. Haygreen, J.G., Bowyer, J.L.: Forest Products and Wood Science: An Introduction, 3rd edn. Iowa State University Press, Ames (1996)

2. Barnett, J.R., Jeronimidis, G.: Wood Quality and its Biological Basis, 1st edn. Blackwell Publishing Ltd., Malden (2003)

3. Ruzin, S.E.: Plant Microtechnique and Microscopy, 1st edn. Oxford University Press, Oxford (1999)

4. Sonka, M., Hlavac, V., Boyle, R.: Ch. 7.2. In: Image Processing, Analysis, and Machine Vision, 3rd edn. Thomson Learning (2008)

5. Xu, C., Prince, J.L.: Snakes, shapes, and gradient vector flow. IEEE Transaction on Image Processing 7(3), 359-369 (1998)

6. Cohen, L.D.: On active contour models and balloons. CVGIP: Image Understanding 53(2), 211-218 (1991)

7. Sonka, M., Hlavac, V., Boyle, R.: Ch. 5.3.5. In: Image Processing, Analysis, and Machine Vision, 3rd edn. Thomson Learning (2008)

8. Kerschner, M.: Homologous twin snakes integrated in a bundle block adjustment. In: International Archives of Photogrammetry and Remote Sensing, vol. XXXII, Part 3/1, pp. 244-249 (1998) 